\title{
Identification of Differentially Expressed Genes in Human Mesenchymal Stem Cell-Derived Neurons
}

\author{
Ji Hye Heo', Kyung Jin Cho', Dal Woong Choi ${ }^{2}$ and Suhng Wook Kim ${ }^{1}$ \\ ${ }^{1}$ Department of Biomedical Science, \\ ${ }^{2}$ Department of Environmental Health, College of Health Sciences, Korea University, Seoul 136-703, Korea
}

(Received January 27, 2010; Revised February 18, 2010; Accepted February 22, 2010)

\begin{abstract}
Mesenchymal stem cells (MSCs) have greater potential for immediate clinical and toxicological applications, due to their ability to self-renew, proliferate, and differentiate into a variety of cell types. To identify novel candidate genes that were specifically expressed during transdifferentiation of human MSCs to neuronal cells, we performed a differential expression analysis with random priming approach using annealing control primer-based differential display reverse transcription-polymerase chain reaction approach. We identified genes for acyl-CoA thioesterase, tissue inhibitor of metalloproteinases-1, brain glycogen phosphorylase, ubiquitin C-terminal hydrolase and aldehyde reductase were up-regualted, whereas genes for transgelin and heparan sulfate proteoglycan were down-regulated in MSC-derived neurons. These differentially expressed genes may have potential role in regulation of neurogenesis. This study could be applied to environmental toxicology in the field of testing the toxicity of a chemical or a physical agent.
\end{abstract}

Key words: Mesenchymal stem cells, Neuron, Differentially expressed genes

\section{INTRODUCTION}

Stem cells are self-renewing unspecialized cells without mature tissue-specific characteristics. Under appropriate conditions, stem cells are able to reproduce themselves and differentiate into many cell types. Stem cells hold tremendous potential in advancing the treatment of many diseases and disorders that are currently untreatable (Korecka et al., 2007; Silani and Corbo, 2004; Kocher et al., 2007; Lock and Tzanakakis, 2007). Embryonic stem cells (ESCs) have vast potential due to their primitive nature and their ability to generate any type of cells (Lerou and Daley, 2005). This pluripotent nature of ESCs makes them attractive for stem cell therapy. However, the disadvantages of ESCs are that they can form teratomas (Lerou and Daley, 2005), and that the use of ESCs poses major ethical concerns.

Adult stem cells are self-renewing and pluripotent cells with a plasticity to differentiate into cell types of the partic-

Correspondence to: Suhng Wook Kim, Department of Biomedical Science, College of Health Sciences, Korea University, San-1, Jeongneung-dong, Seongbuk-gu, Seoul 136-703, Korea

E-mail: swkimkorea@korea.ac.kr

Abbreviations: ESCs, Embryonic stem cells; MSCs, Mesenchymal stem cells; DDRT-PCR, Differential display reverse transcriptionpolymerase chain reaction; ACP, Annealing control primer; FCS, Fetal calf serum; RA, All-trans retinoic acid; bFGF, Basic fibroblast growth factor; TIMP-1, Tissue inhibitor of metalloproteinases-1 ular tissue in which they reside and often to transdifferentiate into different types of tissues (Filip et al., 2004). Adult stem cells are present in many tissues and responsible for renewal and repair of aged or damaged tissue. Adult stem cells show reduced risk for tumorigenesis (Cheng et al., 2004), and their relative ease in harvesting makes the cells appealing, particularly those that reside in the bone marrow.

Mesenchymal stem cells (MSCs) are major adult bone marrow stem cells with multilineage potential (Deans and Moseley, 2002). Furthermore, MSCs are easily obtained from adult bone marrow and can be expanded by simple in vitro procedures (Bianco et al., 2001). Therefore, MSCs have greater potential for immediate clinical and toxicological applications, due to their ability to self-renew, proliferate, and differentiate into a variety of cell types. Bone marrow represents the first source of adult MSCs but other sources have recently been described such as adipose tissue, muscle, brain, and dermis of mammalian skin (Toma et al., 2001; Jiang et al., 2002; Miura et al., 2003; Aust et al., 2004).

Recent literatures have reported the generation of functional neurons transdifferentiated from human MSCs (Cho et al., 2005; Greco et al., 2007). However, little is known about the difference in gene expression during transdifferentiation of MSCs to neuronal cells. The identification of differentially expressed genes induced by transdifferentiation of MSCs to neuronal cells may assist in the identifica- 
tion of potential biomarker and may understand molecular mechanisms during transdifferentiation of MSCs to neuronal cells. Therefore, we performed the differential display reverse transcription-polymerase chain reaction (DDRTPCR) techniques incorporating an annealing control primer (ACP), which has specificity to the template and allows only real products to be amplified (Hwang et al., 2003; Kim et al., 2004), for identification of differentially expressed genes on transdifferentiation of human MSCs to neuronal cells.

\section{MATERIALS AND METHODS}

Culture of human MSCs. Human bone marrow MSCs were purchased from Lonza (Gaithersburg, Maryland, USA). MSCs from passage 5 or 6 were used for experiments and grown in MSC growth medium, DMEM containing 10\% defined fetal calf serum (FCS), which is intended to support MSC maintenance but not differentiation.

Neuronal induction of MSCs. At approximately 70 80\% confluence, MSCs were trypsinized and then subcultured in 60-mm petri dishes. All cells were allowed to adhere to the culture surface overnight in MSC growth medium. At $20 \%$ confluence, MSC growth medium was replaced with neuronal induction medium, which comprised of Ham's DMEM/ F12, 2\% FCS, B27 supplement, $20 \mu \mathrm{M}$ all-trans retinoic acid (RA) and $12.5 \mathrm{ng} / \mathrm{m} l$ basic fibroblast growth factor (bFGF), and cells were cultured as previously described (Cho et al., 2005; Greco et al., 2007). The medium was unchanged during the entire period of induction. In parallel studies, MSCs were cultured in DMEM/F12 and 2\% FCS, hereafter referred to as uninduced MSCs. Experimental endpoints for induced MSCs were 12 days of induction, which correspond to fully differentiated neuronal phenotype.

RT-PCR. Total RNA ( $2 \mu \mathrm{g})$ was reverse transcribed, and 200 ng of cDNA was used to amplify Tac 1 and $\beta$-actin with the AnyDirect Max qPCR Mix (BioQuest, Seoul, Korea). The primers for Tac1 span $+60 /+328$ (GenBank accession number NM_003182) with the following sequences: (forward) 5'-act gtc cgt cgc aaa atc-3' and (reverse) 5'-ggg cca ctt gtt $\mathrm{ttt}$ caa-3'. The primers for $\beta$-actin span $+842 /+1037$ (NM_001101) with the following sequences: (forward) 5'tgc cet gag gca ctc ttc-3' and (reverse) 5'-gtg cca ggg cag tga tct-3'. The cycling profile for for Tac1 and $\beta$-actin (50 cycles) was: $94^{\circ} \mathrm{C}$ for $30 \mathrm{~s}, 60^{\circ} \mathrm{C}$ for $30 \mathrm{~s}$, and $72^{\circ} \mathrm{C}$ for $30 \mathrm{~s}$ with a final extension at $72^{\circ} \mathrm{C}$ for $10 \mathrm{~min}$. The amplified PCR products were separated in $2 \%$ agarose gel and stained with ethidium bromide.

ACP-based DDRT-PCR. Total RNAs extracted from uninduced MSCs and induced MSCs were used for the synthesis of first-strand cDNAs. Reverse transcription was performed for 1.5 hours at $42^{\circ} \mathrm{C}$ in a reaction mixture containing $3 \mu \mathrm{g}$ of the purified total RNA, reaction buffer, $2 \mathrm{mM}$ dNTPs, dT-ACP1 (5'-CGTGAATGCTGCGACTACGATIIIIIT(18)3'), RNase Inhibitor (Promega), and MMLV reverse transcriptase (Promega). Second-strand cDNA was synthesized in a reaction mixture containing first-strand cDNA, $1 \mu l$ of $10 \mu \mathrm{M}$ dT-ACP2 (5'-CGTGAATGCTGCGACTACGATIIIIIT(15)-3'), $10 \mu \mathrm{M}$ arbitrary ACP primers and $2 \times$ Master Mix (Seegene, Seoul, Korea). The PCR protocol for second-strand synthesis was one cycle at $94^{\circ} \mathrm{C}$ for 1 minute, followed by $50^{\circ} \mathrm{C}$ for 3 minutes, and $72^{\circ} \mathrm{C}$ for 1 minute. After second-strand DNA synthesis was completed, the PCR amplification protocol was 40 cycles of $94^{\circ} \mathrm{C}$ for 40 seconds, followed by $65^{\circ} \mathrm{C}$ for 40 seconds, $72^{\circ} \mathrm{C}$ for 40 seconds and 5 minutes final extension at $72^{\circ} \mathrm{C}$. The amplified PCR products were separated in $2 \%$ agarose gel and stained with ethidium bromide. The differentially expressed bands were evaluated based on the band intensities measured with a ChemiDoc XRS (Bio-Rad). The differentially expressed bands were extracted from the gel and directly cloned into a TOPO TA cloning vector (Invitrogen, Carlsbad, CA, USA) according to the manufacturer's instructions. The cloned plasmids were sequenced and the DNA sequence of each gene was confirmed by comparison with sequences in GenBank (NIH, MD, USA).

\section{RESULTS}

Morphology and neuron-specific TAC1 expression of MSCs in neuronal induction medium. To determine whether the induction protocol using neuronal induction medium that included bFGF, RA, B27 could induce MSCs to differentiate into neuronal cells, we first examined morphological changes consistent with neurogenesis. Uninduced MSCs were very flat, symmetrical, and spindle-shaped (Fig. 1A). MSCs induced with neuronal induction medium for 12 days showed a characteristic neuronal morphology with long processes extending from the condensing cell bodies of the induced cells as compared to the symmetric morphologies of the uninduced cells. Cytoplasmic connections between induced cells were also observed (Fig. 1B). Since human MSC-derived neuronal cells have been reported to express the neurotransmitter gene TAC1 (Cho et al., 2005; Greco et al., 2007), we next tested for the expression of the mRNA of TAC1 gene. As shown in Fig. 2, after 12 days of induction, the induced MSCs expressed TAC1 mRNA while uninduced MSCs did not express TAC1 mRNA as determined by RT-PCR and electrophoresis.

Detection of differentially expressed genes. In the aim to determine if the induced MSCs can modify their gene expression profile in response to the neuronal induction medium and to identify novel candidate genes that were specifically expressed, we performed a differential expres- 

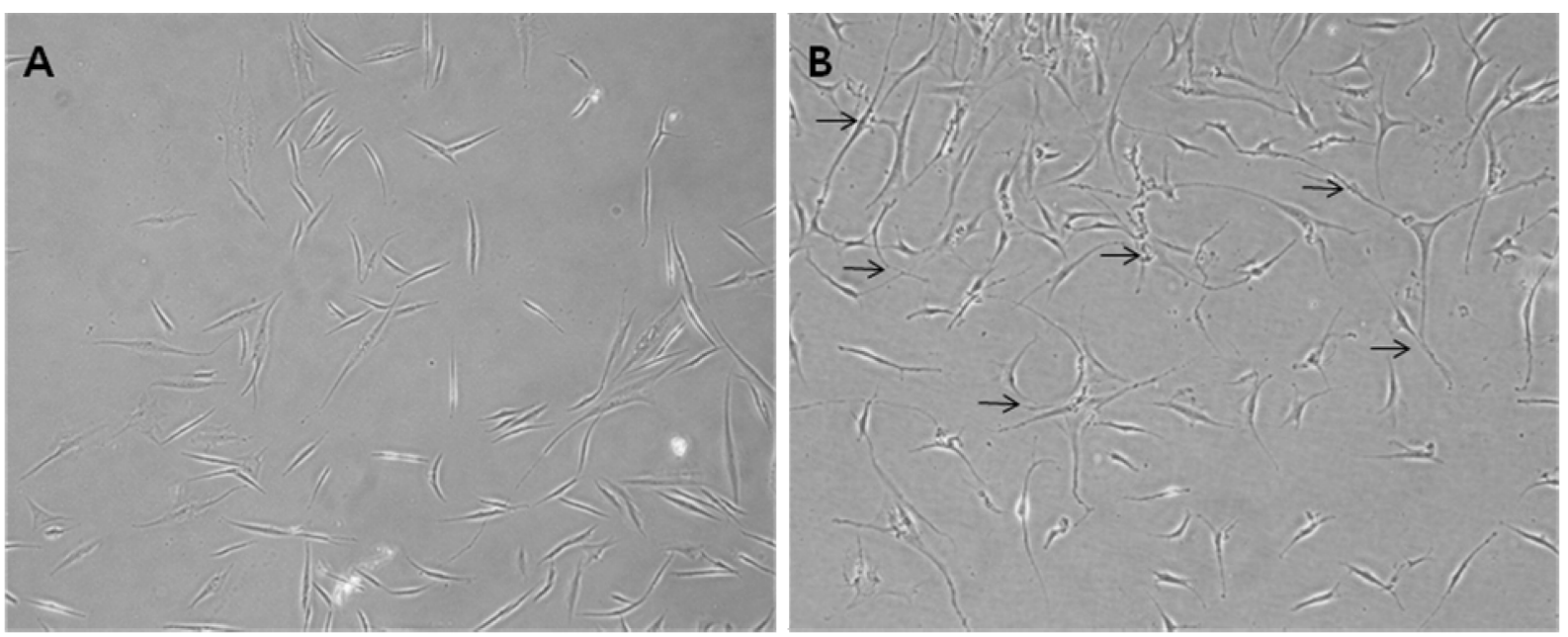

Fig. 1. Morphology of uninduced MSCs (A) and induced MSCs (B). MSCs were induced upon treatment with neuronal induction medium for 12 days. Magnification, A, B $\times 100$. Induced MSCs exhibit long processes with refractive cell bodies. Cytoplasmic connections between induced cells were indicated by arrows.

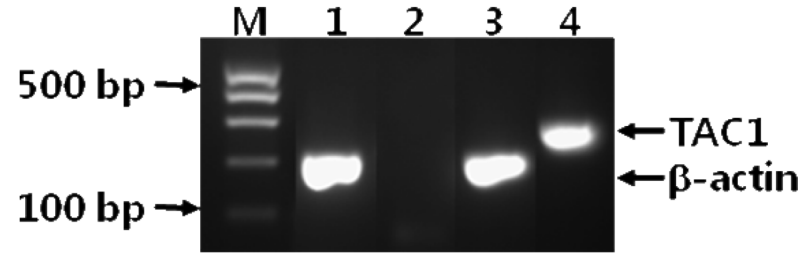

Fig. 2. The expression of neuron-specific TAC1 gene as assessed by RT-PCR. The comparison of the expression patterns of the TAC1 gene in uninduced MSCs (Lanes 1,2) and induced MSCs (Lanes 3 , 4) was determined by semi-quantitative RT-PCR. $\beta$ actin was used as a control to confirm the integrity of the mRNA samples. M, 100-bp DNA ladder.

sion analysis with random priming approach using ACPbased DDRT-PCR between uninduced MSCs and induced MSCs. To do this, the total RNAs from both types of cells were extracted and subjected to ACP-based DDRT-PCR analysis, and the PCR products were loaded on 2\% agarose gels, and upregulated and downregulated genes were identified. When the differentially expressed genes were analyzed by the comparison of band intensities, increased band intensities were detected in ACP1-1 (9\%), ACP2-2 (32\%), ACP6-2 (7\%), ACP9-1 (39\%), ACP10-1 (58\%), ACP10-2 (53\%) and ACP10-3 (51\%), and decreased band intensities were detected in ACP2-1 (26\%), ACP3-1 (39\%), ACP6-1 (54\%) and ACP9-2 (47\%). Fig. 3 shows the results of the screening, which revealed eleven differentially expressed genes indicated by arrows including seven up-regulated and four down-regulated genes in the samples of induced MSC compared with uninduced MSC. These genes were purified, subcloned into TOPO vector and sequenced. BLAST searches in GenBank revealed that the differentially expressed genes displayed significant similarities with known genes. A BLAST search for sequence similarity showed significant similarities (99 100\%) with sequences from Homo sapiens.

Table 1. Sequence similarities of differentially expressed genes

\begin{tabular}{lll}
\hline \hline Clone name* & Identity & $\begin{array}{l}\text { GeneBank } \\
\text { accession no. }\end{array}$ \\
\hline ACP1-1 (up) & Homo sapiens acyl-CoA thioesterase 9 (ACOT9), transcript variant 1, mRNA & NM_001037171 \\
ACP2-1 (down) & Homo sapiens myeloid/lymphoid or mixed-lineage leukemia; translocated to, 6 (MLLT6), mRNA & NM_005937 \\
ACP2-2 (up) & Human interferon-inducible mRNA fragment & X02492 \\
ACP3-1 (down) & Homo sapiens transgelin (TAGLN) gene & EF445034 \\
ACP6-1 (down) & Homo sapiens chromosome 1 clone RP11-496N12 & AC092811 \\
ACP6-2 (up) & Homo sapiens BAC clone RP11-413M20 from 2 & AC074117 \\
ACP9-1 (up) & Homo sapiens TIMP metallopeptidase inhibitor 1 (TIMP1), mRNA & NM_003254 \\
ACP9-2 (down) & Human heparan sulfate proteoglycan (HSPG2) mRNA & M85289 \\
ACP10-1 (up) & Homo sapiens mRNA for brain glycogen phosphorylase variant protein & AB209080 \\
ACP10-2 (up) & Human putative ubiquitin C-terminal hydrolase (UHX1) mRNA & U44839 \\
ACP10-3 (up) & Homo sapiens aldehyde reductase (ALDR1) gene & AF036683 \\
\hline
\end{tabular}

*Up or down in parenthesis means up-regulated genes or down-regulated genes. 


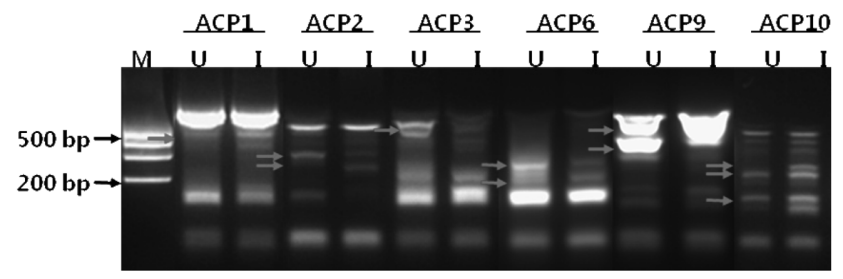

Fig. 3. Detection of differentially expressed genes. Total RNA was extracted from uninduced MSCs and induced MSCs and subjected to ACP-based DDRT-PCR. Ten arbitrary ACP primers (ACP1 to ACP10) were used to isolate the differentially expressed genes. Differential expression patterns were observed when the arbitrary ACP primer sets (indicated on the top) were used. The differential expression patterns were evaluated based on the band intensities. The arrows on the left-hand side indicate differential expressed bands between uninduced MSCS (U) and induced MSCs (I). Bands were excised from the gel for sequencing. $M, 100-b p$ DNA ladder.

The sequence similarities of differentially expressed genes are summarized in Table 1.

\section{DISCUSSION}

MSCs represent attractive candidates for a wide range of regenerative medicine applications due to their relative ease in harvesting, isolation and expansion and the important potential of differentiation. However, molecular mechanisms implied in the differentiation process remain largely unknown. The difference in gene expression on transdifferentiation of human MSCs to neuronal cells must be good targets to identify the genetic factors associated with the differentiation process.

Gene expression profiling can be achieved by several approaches, each with inherent advantages and disadvantages. In this study, we used ACP-based DDRT-PCR approach, which was recently developed to allow direct visualization of the differentially expressed transcripts on agarose gel. This technology improves the specificity and sensitivity of PCR amplification so that it enables us to find only authentic PCR products. Using the random priming ACP-based DDRT-PCR approach for differential expression profiling, we have successfully identified seven upregulated and four downregulated genes in induced MSCs. We identified genes for acyl-CoA thioesterase, tissue inhibitor of metalloproteinases-1, brain glycogen phosphorylase, ubiquitin C-terminal hydrolase and aldehyde reductase were up-regualted, whereas genes for transgelin and heparan sulfate proteoglycan were down-regulated in induced MSCs. Some of these differentially expressed genes were found to be neuron related.

Acyl-CoA thioesterase catalyzes the hydrolysis of CoA esters to free CoA and carboxylic acids and have important functions in lipid metabolism and other cellular processes.
Acyl-CoA thioesterase expression was found to be induced during embryogenesis in association with neuronal differentiation, and persists after terminal differentiation into neurons in postnatal stages, resulting in the constitutive high expression of Acyl-CoA thioesterase in the adult brain in a neuron-specific manner (Yamada et al., 2004). Tissue inhibitor of metalloproteinases-1 (TIMP-1) displays inhibitory activity on matrix metalloproteinases that degrade the extracellular matrix and shed cell surface molecules. In the central nervous system, TIMP-1 was first characterized as a candidate plasticity gene induced by seizures and by stimuli leading to long term potentiation (Nedivi et al., 1993), a form of synaptic plasticity considered as a cellular substrate of learning and memory. Recently, TIMP-1 was found to be a modulator of neuronal outgrowth and morphology in a paracrine and autrocrine manner through the inhibition of matrix metalloproteinases (Ould-yahoui et al., 2009). Ubiquitin C-terminal hydrolase is also known as neuronal-specific protein gene product 9.5 and was previously used as a histologic marker for neurons because of its high abundance and specific expression in neurons. Ubiquitin C-terminal hydrolase is present in almost all neurons and averages $1 \sim$ $5 \%$ of total soluble brain protein (Jackson and Thompson, 1981). These differentially expressed genes may have potential role in regulation of neurogenesis. However, the direct relevance of these differentially expressed genes in MSC differentiation has not been elucidated. Further studies are needed to validate these findings.

Another potential of the study of MSC differentiation is in the field of testing the toxicity of a chemical or a physical agent. The primary benefits of MSCs when applied to environmental toxicology, result not only from their unique properties of proliferative ability, but also plasticity to generate other cell types (Davila et al., 2004). The rapid accumulation of genomic sequence data have catalyzed the application of gene expression analysis in understanding the mode of action of chemical and other environmental toxic agent in biological systems. Treating MSC with chemical or physical agents and measuring their gene expression levels can, not only provide information about the mechanism of action of toxicants, but also a gene expression patterns. The development of such gene expression patterns would allow the screening of unknown or suspected toxicants on the basis of their similarity to known toxicants.

\section{ACKNOWLEDGMENTS}

This work was supported by the Health Fellowship Foundation.

\section{REFERENCES}

Aust, L., Devlin, B., Foster, S.J., Halvorsen, Y.D., Hicok, K., du Laney, T., Sen, A., Willingmyre, G.D. and Gimble, J.M. (2004). 
Yield of human adipose-derived adult stem cells from liposuction aspirates. Cytotherapy, 6, 7-14.

Bianco, P., Riminucci, M., Gronthos, S. and Robey, P.G. (2001). Bone marrow stromal stem cells: nature, biology, and potential applications. Stem Cells, 19, 180-192.

Cheng, N., Janumyan, Y.M., Didion, L., Van Hofwegen, C., Yang, E. and Knudson, C.M. (2004). Bcl-2 inhibition of T-cell proliferation is related to prolonged T-cell survival. Oncogene, 23, 3770-3780

Cho, K.J., Trzaska, K.A., Greco, S.J., McArdle, J., Wang, F.S., Ye, J.H. and Rameshwar, P. (2005). Neurons derived from human mesenchymal stem cells show synaptic transmission and can be induced to produce the neurotransmitter substance $\mathrm{P}$ by interleukin-1 alpha. Stem Cells, 23, 383-391.

Davila, J.C., Cezar, G.G., Thiede, M., Strom, S., Miki, T. and Trosko, J. (2004). Use and application of stem cells in toxicology. Toxicol. Sci., 79, 214-223.

Deans, R.J. and Moseley, A.B. (2000). Mesenchymal stem cells: biology and potential clinical uses. Exp. Hematol., 28, 875-884.

Filip, S., English, D. and Mokry, J. (2004). Issues in stem cell plasticity. J. Cell. Mol. Med., 8, 572-577.

Greco, S.J., Zhou, C., Ye, J.H. and Rameshwar, P. (2007). An interdisciplinary approach and characterization of neuronal cells transdifferentiated from human mesenchymal stem cells. Stem Cells Dev., 16, 811-826.

Hwang, I.T., Kim, Y.J., Kim, S.H., Kwak, C.I., Gu, Y.Y. and Chun, J.Y. (2003). Annealing control primer system for improving specificity of PCR amplification. BioTechniques, 35, 11801184.

Jackson, P. and Thompson, R.J. (1981). The demonstration of new human brain-specific proteins by high-resolution two-dimensional polyacrylamide gel electrophoresis. J. Neurol. Sci., 49, 429-438.

Jiang, Y., Jahagirdar, B.N., Reinhardt, R.L., Schwartz, R.E., Keene, C.D., Ortiz-Gonzalez, X.R., Reyes, M., Lenvik, T., Lund, T., Blackstad, M., Du, J., Aldrich, S., Lisberg, A., Low, W.C., Largaespada, D.A. and Verfaillie, C.M. (2002). Pluripotency of mesenchymal stem cells derived from adult marrow.
Nature, 418, 41-49.

Kim, Y.J., Kwak, C.I., Gu, Y.Y., Hwang, I.T. and Chun, J.Y. (2004). Annealing control primer system for identification of differentially expressed genes on agarose gels. BioTechniques, 36, 424-430.

Kocher, A.A., Schlechta, B., Gasparovicova, A., Wolner, E., Bonaros, N. and Laufer, G. (2007). Stem cells and cardiac regeneration. Transpl. Int., 20, 731-746.

Korecka, J.A., Verhaagen, J. and Hol, E.M. (2007). Cell-replacement and gene-therapy strategies for Parkinson's and Alzheimer's disease. Regen. Med., 2, 425-446.

Lerou, P.H. and Daley, G.Q. (2005). Therapeutic potential of embryonic stem cells. Blood Rev., 19, 321-331.

Lock, L.T. and Tzanakakis, E.S. (2007). Stem/Progenitor cell sources of insulin-producing cells for the treatment of diabetes. Tissue Eng., 13, 1399-1412.

Miura, M., Gronthos, S., Zhao, M., Lu, B., Fisher, L.W., Robey, P.G. and Shi, S. (2003). SHED: stem cells from human exfoliated deciduous teeth. Proc. Natl. Acad. Sci. U.S.A., 100, 58075812.

Nedivi, E., Hevroni, D., Naot, D., Israeli, D. and Citri, Y. (1993). Numerous candidate plasticity-related genes revealed by differential cDNA cloning. Nature, 363, 718-722.

Ould-yahoui, A., Tremblay, E., Sbai, O., Ferhat, L., Bernard, A., Charrat, E., Gueye, Y., Lim, N.H., Brew, K., Risso, J.J., Dive, V., Khrestchatisky, M. and Rivera, S. (2009). A new role for TIMP-1 in modulating neurite outgrowth and morphology of cortical neurons. PLoS One, 4, e8289.

Silani, V. and Corbo, M. (2004). Cell-replacement therapy with stem cells in neurodegenerative diseases. Curr. Neurovasc Res., 1, 283-289.

Toma, J.G., Akhavan, M., Fernandes, K.J., Barnabe-Heider, F., Sadikot, A., Kaplan, D.R. and Miller, F.D. (2001). Isolation of multipotent adult stem cells from the dermis of mammalian skin. Nat. Cell Biol., 3, 778-784.

Yamada, J., Kuramochi, Y., Takagi, M. and Suga, T. (2004). Expression of acyl-CoA hydrolase in the developing mouse brain. Neurosci. Lett., 355, 89-92. 